\title{
Buprenorphine Dispensing in Pennsylvania During the COVID-19 Pandemic, January to October 2020
}

J Gen Intern Med 36(12):3915-7

DOI: $10.1007 / \mathrm{s} 11606-021-07083-y$

(c) Society of General Internal Medicine 2021

\section{INTRODUCTION}

The COVID-19 pandemic created unique challenges for treatment of opioid use disorders (OUD). Opioid overdoses have increased, ${ }^{1}$ even as restrictions on prescribing of buprenorphine - used to treat OUD — have been eased by expanded access via telemedicine. Studies have examined shifts in buprenorphine prescribing during the early pandemic, ${ }^{2-5}$ but findings about initiation of buprenorphine treatment-a key parameter in assessing disruption of care-are conflicting. A study of Texas Prescription Drug Monitoring Program (PDMP) data found a marked increase in new buprenorphine patients in the first 90 days of the pandemic, ${ }^{2}$ while a study using Optum commercial data found a decrease in new buprenorphine patients. ${ }^{3}$ These studies, however, extend only $2^{3}$ to $3^{2}$ months into the pandemic. Moreover, studies using commercial insurance data miss key populations that do not have, or that lose, commercial insurance..$^{3-5}$

To examine these trends over a longer time period and among all patients filling buprenorphine, we used all-payer controlled substance prescription data from Pennsylvania to examine changes in outpatient buprenorphine dispensing after the start of the pandemic, using data from January to October 2020 compared to a similar time period in 2019 .

\section{METHODS}

We analyzed data from the Pennsylvania PDMP, which collects information on all controlled substance prescription fills in Pennsylvania, regardless of payment type. We identified all outpatient fills for buprenorphine products (excluding transdermal buprenorphine for pain) from January to October in both 2019 and 2020. Among these products, approximately $83 \%$ were buprenorphine combined with naloxone.

Each week, we calculated the total number of prescriptions filled and number of unique patients filling buprenorphine. We also calculated the number of buprenorphine-naïve patients (defined as not having filled a prescription in the

Received June 1, 2021

Accepted July 21, 2021

Published online August 10, 2021 previous 180 days [or 45 days as sensitivity analysis]) filling buprenorphine prescriptions each week. Finally, to parse the effect of the length of prescriptions on overall buprenorphine dispensing, we examined weekly changes in the days' supply of buprenorphine prescriptions and the number of patients with active buprenorphine prescriptions (the number of patients with at least 1 day of supply available in a given week).

For each parameter, we performed comparative interrupted time series regression to compare trends in dispensing in 2019 and 2020 before and after the pandemic started. We defined pandemic start at calendar week 12, beginning March 22, 2020 (see the figure footnote for details). In a sensitivity analysis, we excluded week 11 as a transition period.

\section{RESULTS}

In 2020, the weekly number of patients filling buprenorphine prescriptions significantly decreased after week 12 , compared to the same period in $2019(-1106$ patients per week [95\% CI: $-1470,-741], P<0.01$; Fig. 1A, Table 1). The weekly number of buprenorphine-naïve patients filling buprenorphine prescriptions also significantly decreased $(-297$ patients per week [95\% CI: $-347,-247$ ], $P<0.01$; Fig. 1B, Table 1), with an increase back to 2019 levels by August. The reduction in buprenorphine prescriptions was accompanied by an increase in the mean days' supply ( +0.65 days' supply [95\% CI: 0.38 , 0.92], $P<0.01$; Fig. 1C, Table 1). Overall, there was no statistically significant change in patients with an active buprenorphine prescription $(-167$ patients per week [95\% CI: -543, 209]), although the rate of growth after week 12 decreased compared to the same period in 2019 ( -52 patients per week [95\% CI: $-71,-33], P<0.01$; Fig. 1D, Table 1). Results of sensitivity analyses excluding week 11 were similar.

\section{DISCUSSION}

The onset of the COVID-19 pandemic in Pennsylvania was associated with a reduction in both the number of patients filling buprenorphine prescriptions and new patients initiating buprenorphine. These changes, however, were accompanied by an increase in mean days' supply and no overall change in the number of patients with active buprenorphine prescriptions.

Although our study was limited to Pennsylvania and only captures buprenorphine dispensed by pharmacies, it captures all payers and expands upon the time period of previous 
A: Patients filling prescriptions for buprenorphine each week

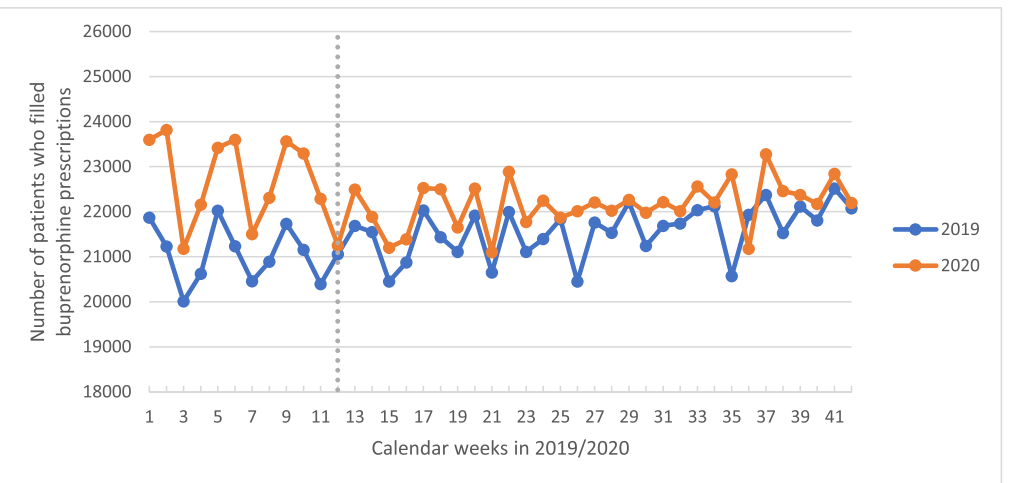

B: Buprenorphine-naïve patients filling prescriptions for buprenorphine

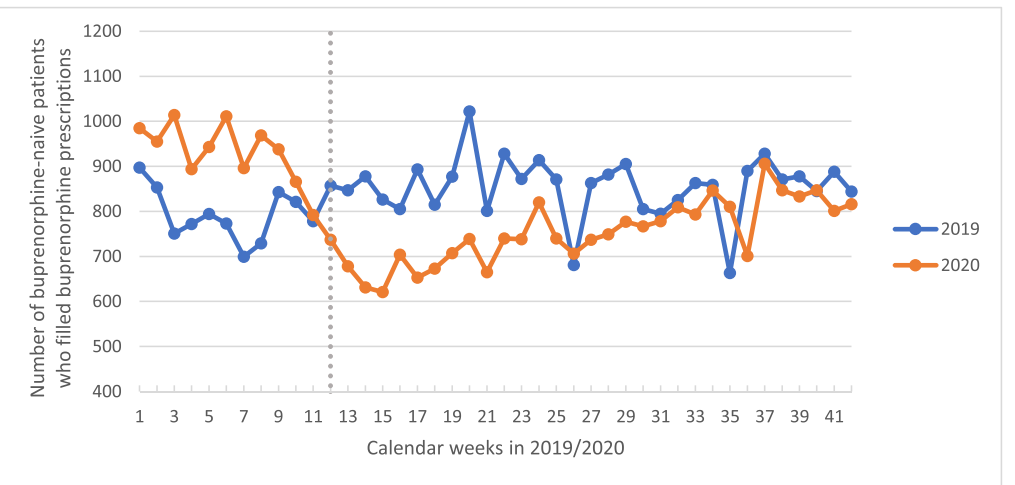

C: Mean days' supply of filled buprenorphine prescriptions

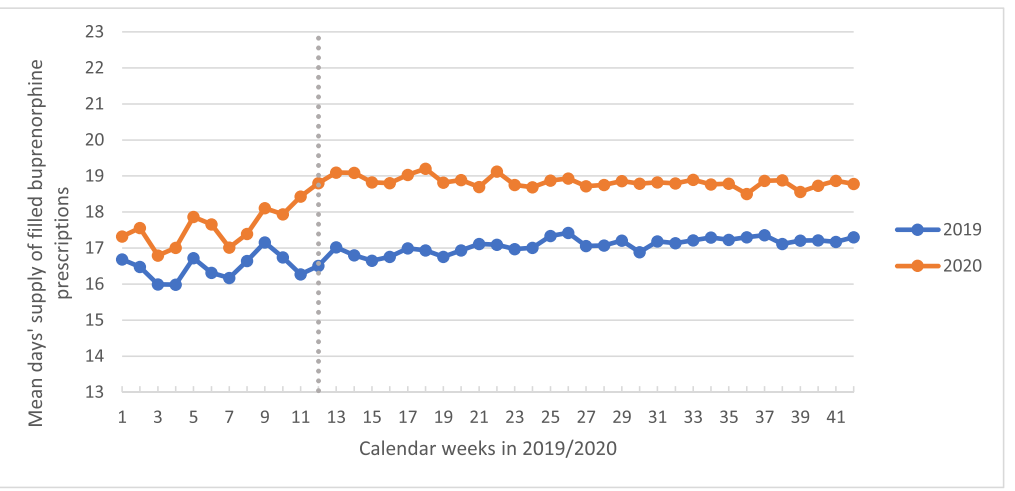

D: Patients with active prescriptions for buprenorphine each week

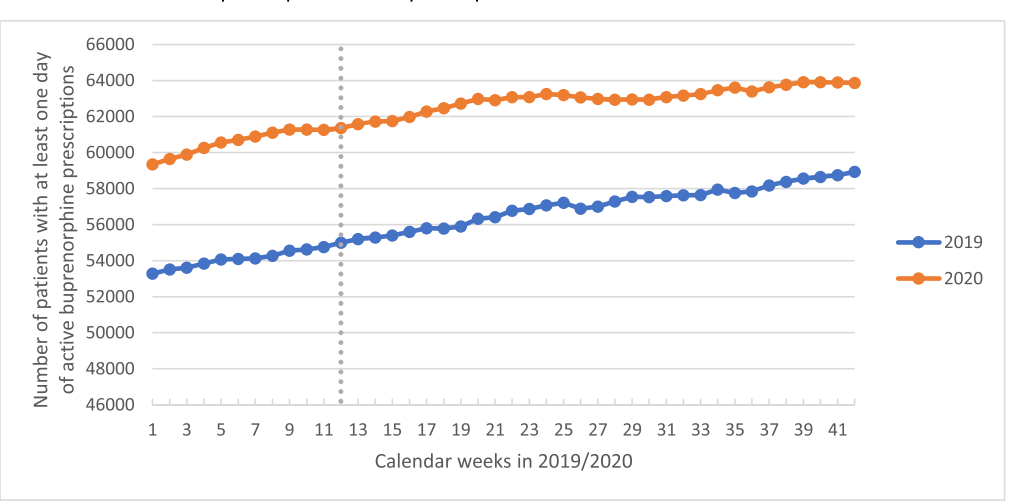

Fig. 1 Pennsylvania buprenorphine dispensing in January-October, 2019 and 2020. A Patients filling prescriptions for buprenorphine each week. B Buprenorphine-nä̈ve patients filling prescriptions for buprenorphine. C Mean days' supply of filled buprenorphine prescriptions. D Patients with active prescriptions for buprenorphine each week. For each figure, we used the beginning of calendar week 12 (March 22, 2020 ) as the transition between analysis periods (corresponding to the date Pennsylvania instituted stay-at-home orders in major counties because of COVID; 2 weeks after COVID-19 was declared an emergency in Pennsylvania; 1 week before restrictions around telehealth for medicationassisted treatment services were eased). The transition is indicated in each panel by a dotted vertical line at week 12. 
Table 1 Changes in Buprenorphine Fill Patterns, January to October 2019 and 2020

\begin{tabular}{|c|c|c|c|c|c|c|c|c|}
\hline & $\begin{array}{l}\text { Patients filling } \\
\text { buprenorphine } \\
\text { prescriptions } \\
(95 \% \text { CI) }\end{array}$ & $\begin{array}{l}P \\
\text { value }\end{array}$ & $\begin{array}{l}\text { Buprenorphine- } \\
\text { naïve patients } \\
\text { (95\% CI) }\end{array}$ & $\begin{array}{l}P \\
\text { value }\end{array}$ & $\begin{array}{l}\text { Mean days' } \\
\text { supply } \\
(95 \% \text { CI) }\end{array}$ & $\begin{array}{l}P \\
\text { value }\end{array}$ & $\begin{array}{l}\text { Patients with } \\
\text { active } \\
\text { buprenorphine } \\
\text { prescriptions } \\
(95 \% \text { CI) }\end{array}$ & $\begin{array}{l}P \\
\text { value }\end{array}$ \\
\hline \multicolumn{9}{|c|}{ Change at transition } \\
\hline 2019 & $\begin{array}{l}267.7 \\
(53.6,481.7)\end{array}$ & 0.02 & $\begin{array}{l}103.3 \\
(64.5,142.1)\end{array}$ & $<0.001$ & $\begin{array}{l}0.16 \\
(-0.01,0.34)\end{array}$ & 0.07 & $\begin{array}{l}284.7 \\
(109.3,460.1)\end{array}$ & 0.002 \\
\hline 2020 & $\begin{array}{l}-838.1(-1133.0 \\
-543.2)\end{array}$ & $<0.001$ & $\begin{array}{l}-193.5 \\
(-224.6,-162.5)\end{array}$ & $<0.001$ & $\begin{array}{l}0.81 \\
(0.61,1.01)\end{array}$ & $<0.001$ & $\begin{array}{l}118.1 \\
(-214.6,450.7)\end{array}$ & 0.48 \\
\hline Difference & $\begin{array}{l}-1105.8(-1470.2 \\
-741.4)\end{array}$ & $<0.001$ & $\begin{array}{l}-296.8 \\
(-346.5,-247.1)\end{array}$ & $<0.001$ & $\begin{array}{l}0.65 \\
(0.38,0.92)\end{array}$ & $<0.001$ & $\begin{array}{l}-166.7 \\
(-542.7,209.4)\end{array}$ & 0.38 \\
\hline \multicolumn{9}{|c|}{$\begin{array}{l}\text { Post-intervention weekly } \\
\text { growth rate }\end{array}$} \\
\hline 2019 & $\begin{array}{l}29.5 \\
(22.4,36.6)\end{array}$ & $<0.001$ & $\begin{array}{l}-0.4 \\
(-1.4,0.6)\end{array}$ & 0.40 & $\begin{array}{l}0.018 \\
(0.012 \\
0.023)\end{array}$ & $<0.001$ & $\begin{array}{l}123.6 \\
(113.6,133.6)\end{array}$ & $<0.001$ \\
\hline 2020 & $\begin{array}{l}23.2 \\
(18.9,27.4)\end{array}$ & $<0.001$ & $\begin{array}{l}6.2 \\
(5.4,7.0)\end{array}$ & $<0.001$ & $\begin{array}{l}-0.008 \\
(-0.010 \\
-0.006)\end{array}$ & $<0.001$ & $\begin{array}{l}71.6 \\
(55.9,87.3)\end{array}$ & $<0.001$ \\
\hline Difference & $\begin{array}{l}-6.3 \\
(-14.6,1.9)\end{array}$ & 0.13 & $\begin{array}{l}6.6 \\
(5.4,7.9)\end{array}$ & $<0.001$ & $\begin{array}{l}-0.026 \\
(-0.032 \\
-0.020)\end{array}$ & $<0.001$ & $\begin{array}{l}-52.0 \\
(-70.6,-33.4)\end{array}$ & $<0.001$ \\
\hline
\end{tabular}

The change at transition in 2019 and 2020 represents the immediate change at week 12 in the regression model (e.g., change in the weekly number of patients filling prescriptions for buprenorphine). The difference in the change at transition is the difference between the 2019 and 2020 changes. The post-intervention weekly growth rates in 2019 and 2020 are determined from regression coefficients for the treatment (2020) and control (2019) groups in the post-intervention linear time trends from the comparative interrupted time series analysis. The difference in post-intervention weekly growth rates is the difference between the 2019 and 2020 coefficients

analyses, with data through October. The observed reduction in new initiation of buprenorphine has major clinical and public health importance, highlighting a deficit in treatment of OUD created by the pandemic and response, despite implementation of policies easing prescribing restrictions nationwide. Continuing to ease the ability to initiate buprenorphine through telemedicine or increasing the number of prescribers who can start therapy could attenuate the clinical impacts of these kinds of disruptions.

Rohan Chalasani, $B A^{1,2}$

Jared M. Shinabery, $M P H^{3}$

Carrie Thomas Goetz, $P h D^{3}$

Chung-Chou H. Chang, $\mathrm{PhD}^{4}$

Qingnan Yang, MS, $M A^{2}$

Katie J. Suda, PharmD, MS, FCCP $P^{2,4}$

Walid F. Gellad, MD, MPH ${ }^{2,4}$

${ }^{1}$ Feinberg School of Medicine, Northwestern University,

Chicago, IL, USA

${ }^{2}$ Center for Pharmaceutical Policy and Prescribing, Health Policy Institute, University of Pittsburgh,

Pittsburgh, PA, USA

${ }^{3}$ Drug Surveillance and Misuse Prevention Office, Pennsylvania Department of Health,

Harrisburg, PA, USA

${ }^{4}$ Division of General Internal Medicine, School of Medicine, University of Pittsburgh,

Pittsburgh, PA, USA

Corresponding Author: Walid F. Gellad, MD, MPH; Division of General Internal Medicine, School of Medicine, University of Pittsburgh, Pittsburgh, PA, USA (e-mail: walid.gellad@pitt.edu).
Funding This analysis was supported by a contract between the Pennsylvania Department of Health and the University of Pittsburgh, funded by the Centers for Disease Control and Prevention of the US Department of Health and Human Services (HHS) as part of a financial assistance award with 100\% funded by CDC/HHS (Grant No. 1 NU17CE924973-01-00). The views expressed are those of the author(s) and do not necessarily represent the official views of, nor an endorsement, by CDC/HHS, or the US Government.

\section{Declarations:}

Conflict of Interest: The authors declare that they do not have a conflict of interest.

\section{REFERENCES}

1. Ochalek TA, Cumpston KL, Wills BK, Gal TS, Moeller FG. Nonfatal opioid overdoses at an urban emergency department during the COVID-19 pandemic. JAMA. 2020; 324(16): 1673-1674.

2. Cance JD, Doyle E. Changes in outpatient buprenorphine dispensing during the COVID-19 pandemic. JAMA. 2020; 324(23): 2442-2444.

3. Huskamp HA, Busch AB, Uscher-Pines L, Barnett ML, Riedel L, Mehrotra A. Treatment of opioid use disorder among commercially insured patients in the context of the COVID-19 pandemic. JAMA. 2020; 324(23): 24402442 .

4. Nguyen TD, Gupta S, Ziedan E, Simon KI, Alexander GC, Saloner B, Stein BD. Assessment of filled buprenorphine prescriptions for opioid use disorder during the coronavirus disease 2019 pandemic. JAMA Internal Medicine. 2020; published online.

5. Jones CM, Guy Jr GP, Board A. Comparing actual and forecasted numbers of unique patients dispensed select medications for opioid use disorder, opioid overdose reversal, and mental health, during the COVID-19 pandemic, United States, January 2019 to May 2020. Drug and alcohol dependence. 2021; 219: 108486.

Publisher's Note: Springer Nature remains neutral with regard to jurisdictional claims in published maps and institutional affiliations. 